\title{
La formation des étudiants marocains dans les pays de l'Est de l'Europe (1960-2015)
}

Moroccan Students' Training in Eastern Europe Countries (1960-2015)

La formación de los estudiantes marroquíes en los países de Europa del Este

(1960-2015)

Kamal Mellakh

\section{(2) OpenEdition}

Journals

Édition électronique

URL : https://journals.openedition.org/remi/7777

DOI : $10.4000 /$ remi. 7777

ISSN : $1777-5418$

Éditeur

Université de Poitiers

Édition imprimée

Date de publication : 1 septembre 2016

Pagination : 39-56

ISBN : 979-10-90426-27-6

ISSN : 0765-0752

Référence électronique

Kamal Mellakh, «La formation des étudiants marocains dans les pays de l'Est de l'Europe

(1960-2015) », Revue européenne des migrations internationales [En ligne], vol. 32 - n² 2 2016, mis en ligne le 01 septembre 2018, consulté le 14 avril 2022. URL : http://journals.openedition.org/remi/7777 ; DOI : https://doi.org/10.4000/remi.7777 


\section{La formation des étudiants marocains dans les pays de l'Est de l'Europe (1960-2015)}

\section{Kamal Mellakh ${ }^{1}$}

Les mobilités des étudiants marocains vers les pays de l'Est de l'Europe remontent au moins au début des années 1960. Mais elles se sont déroulées dans l'ombre de celles, plus massives, vers la France, si bien qu'elles furent longtemps ignorées et peu étudiées. Si l'État marocain s'est activé dès l'indépendance en 1956 pour mettre en place des filières de mobilité étudiante vers I'ancien pays colonisateur, force est de constater que les étudiants marocains et leurs familles ont été plus opportunistes en investissant les possibilités de formation offertes par d'autres pays comme I'URSS et les anciens pays socialistes (Roumanie, Pologne, Tchécoslovaquie, etc.). Les premiers départs vers I'Union Soviétique ont lieu en dehors des circuits habituels de la coopération d'État à État. Mais à partir des années 1960, l'établissement d'accords de coopération a donné une assise institutionnelle à ces mobilités anciennes et particulières dans le paysage migratoire marocain. Jusqu'à la fin des années 1980, les départs ont utilisé plusieurs circuits : le ministère, les partis politiques, les ambassades. L'obtention d'une bourse fut souvent ce qui a déclenché les projets de départ. Mais après 1991, et le passage à l'ère post-soviétique, une rupture s'est opérée avec le recul des offres de bourses par l'État et l'augmentation des formations payantes. Les possibilités de départ concernent alors plus les étudiants issus des classes moyennes qui peuvent supporter le coût des études dans les filières médicales et paramédicales. Les départs individuels et spontanés supportés financièrement par les familles se sont intensifiés. Comment comprendre la persistance des départs d'étudiants marocains pour ces formations dans des pays lointains avec lesquels le Maroc n'avait pas de " proximité éducative " ? Comment se sont construits ces projets migratoires ? Comment ces mobilités se sont maintenues au fil du temps et comment elles se sont transformées à l'ère post-soviétique ?

Les pays de l'Est ne forment pas un tout homogène et il sera difficile, en l'état actuel de notre recherche, d'apporter à ces questions des réponses exhaustives prenant en compte les logiques et les temporalités singulières de chaque pays (Russie, Ukraine, Roumanie, Bulgarie, etc.). Cette contribution propose de fournir un aperçu historique sur ces mobilités et de donner des éléments de

1 Enseignant-chercheur, Département de sociologie, Faculté des lettres et sciences humaines de Mohammedia, Université Hassan II, Casablanca B.P 546, Mohammedia, Maroc ; kmellakh@yahoo.fr 
compréhension du nouveau contexte migratoire qui les favorise. L'intérêt des étudiants marocains et de leurs familles pour les études dans les pays de l'Est va grandissant. Étudier ces mobilités, c'est également rendre compte des dynamiques socio-éducatives qui contribuent aujourd'hui au Maroc à faire de l'Est de l'Europe une destination prisée au sein du système de mobilité internationale des étudiants marocains.

Notre analyse prend appui sur des entretiens réalisés auprès d'anciens diplômés partis se former en URSS/Russie à différentes époques ${ }^{2}$, sur les statistiques de I'UNESCO et sur les données des organisations marocaines (I'Association Marocaine des Lauréats des Instituts et Universités Soviétiques - AMLIUS et l'Ordre National des Pharmaciens du Maroc).

\section{Ouverture de la filière soviétique de formation (1960-1966) : entre méfiance et tolérance de l'État}

Les départs des étudiants marocains vers I'URSS ont augmenté d'une manière significative au début des années 1960, lorsque les relations diplomatiques entre le Maroc et I'URSS ont connu un nouvel essor transcendant les divergences idéologiques des deux régimes politiques. Les leaders historiques de I'indépendance, conservateurs (Allal Al Fassi) ${ }^{3}$ ou progressistes (Mehdi Ben Barka $)^{4}$, ont poussé la monarchie marocaine à opter, en pleine Guerre froide, pour un neutralisme politique et une ouverture vers les pays socialistes, afin de se dégager pour partie de la tutelle occidentale (notamment de la France) et de confirmer le nouveau statut du "Maroc indépendant " (Berramdane, 1987 : 163-189). Cette orientation de la politique extérieure marocaine a favorisé l'établissement d'échanges éducatifs entre le Maroc et I'URSS dès la fin des années 1950. Par exemple, le 13 juin 1960, I'ambassadeur soviétique à Rabat publie une note réitérant l'offre de bourses soviétiques aux étudiants marocains. Et en août 1962 une délégation de I'UNEM (Union Nationale des Étudiants du Maroc) part visiter Moscou (Berramdane, 1987 : 182). Toutefois, il convient de souligner qu'en dépit de l'existence d'un rapprochement entre les deux États couronné par la signature de plusieurs accords diplomatiques de coopération ${ }^{5}$, les premiers départs d'étudiants ont eu lieu en dehors du circuit habituel de la

\footnotetext{
2 Nous avons mené quinze entretiens semi-directifs auprès des anciens diplômés formés en URSS/Russie : quatre entretiens auprès de diplômés partis avant le démantèlement de I'URSS et onze entretiens avec des diplômés partis postérieurement. Pour la période soviétique, nous avons mené deux entretiens avec des pharmaciens partis respectivement en 1970 et en 1988, un entretien avec un architecte parti en 1960 et un entretien avec un enseignant-chercheur de la faculté des lettres et sciences humaines de Rabat, aujourd'hui retraité, parti en 1961. Les entretiens réalisés avec les diplômés partis durant la période post soviétique (après 1991) sont répartis comme suit : neuf entretiens avec des pharmaciens et deux entretiens avec des médecins.

3 Allal Al Fassi (1906-1974) est I'un des principaux leaders du mouvement national de lutte contre l'indépendance. II est connu par sa pensée politique et sociale conservatrice.

4 Mehdi Ben Berka (1920-1965) est un des chefs de file de l'aile progressiste du mouvement national. II était le leader historique de la gauche marocaine.

5 Le premier accord de coopération entre le Maroc et I'URSS était du type commercial. Il a été signé en avril 1957, soit juste une année après l'indépendance du pays. L'établissement des relations diplomatiques date de 1958. C'est en 1966 que le premier accord global de coopération économique, technique et culturelle fut signé entre les deux parties (Berramdane, 1987).
} 
coopération d'État à État. Ils sont partis au début des années 1960 grâce à des bourses soviétiques obtenues directement auprès des partis politiques, des syndicats, de I'Union Nationale des Étudiants du Maroc qui était proche à cette époque de la gauche marocaine, notamment de I'Union Socialiste des Forces populaires ou du mouvement marxiste-léniniste Ila al Amam. Les étudiants n'avaient nullement besoin de passer par les services de l'État pour partir ; tel fut le cas par exemple de l'Architecte Omar Sbai, diplômé de I'Institut d'Architecture de Moscou en 1968. Après un baccalauréat "Sciences expérimentales " obtenu en 1959 à Rabat, il s'est rendu à Moscou en 1960 grâce à une bourse du parti de I'Istiqlal ${ }^{6}$ :

" Je suis parti en 1960 à Moscou. C'était un pur hasard, car je n'avais jamais pensé avant, que j'allais étudier en URSS ou dans un autre pays étranger. À l'époque, j'étais membre de l'association de scoutisme Hassania qui était très proche du parti de l'Istiqlal. Notre commandant a été au courant que je venais juste d'avoir le bac. II m'a demandé si je voulais poursuivre mes études à l'étranger et notamment en URSS. C'est comme ça que les choses ont commencé. J'étais parmi un groupe de onze bacheliers qui ont pu partir en 1960 grâce à une bourse de parti de l'Istiqlal. Lorsque je suis arrivé à Moscou, il y avait déjà quelques étudiants marocains. En 1963-1964, nous étions au moins trente-cinq étudiants marocains à Moscou " (Omar Sbai).

Ce n'est qu'après l'établissement du premier accord de coopération économique et technique en 1966 que les autorités marocaines ont commencé à prendre en charge les départs des étudiants vers I'URSS. Jusque-là, le départ des étudiants échappait au contrôle de l'État, ce qui a d'ailleurs amené le gouvernement marocain à protester contre la politique de gestion des bourses de I'URSS (Katsakioris, 2007 : 94). II faut dire que les autorités marocaines étaient à la fois favorables et sceptiques face à l'offre éducative soviétique. Si elles étaient favorables à la formation des cadres dans les pays socialistes pour les besoins de reconstruction du pays (Scarfò, 2015 : 190), elles étaient par contre méfiantes face à la formation dispensée dans les pays communistes sans aucune forme de contrôle. L'État Marocain a ainsi cherché dès les années 1960 à établir des accords de coopération avec Moscou et les autres capitales de ce qu'on appelait "le bloc soviétique " pour profiter de l'aide apportée, mais aussi pour contrôler le flux de cadres marocains formés dans les pays communistes et susceptibles de rejoindre les mouvements de gauche avec lesquels la monarchie était en confrontation directe tout au long des années 1960 et 1970. II est vrai que les premiers étudiants partis en URSS au début des années 1960 appartenaient à cette première génération d'étudiants marocains à l'étranger, impliqués d'une manière ou d'une autre dans les luttes politiques et syndicales de l'époque (Laffort, 2009). Le parcours d'Abderrahim El Attaoui, professeur de langue et littérature russe à la faculté des lettres et des sciences humaines de Rabat, diplômé de la faculté de philologie de l'université de Leningrad en 1967, est significatif à cet égard. II est parti en 1961 avec une bourse soviétique obtenue grâce au mouvement de la jeunesse ouvrière affilié à I'Union Marocaine du Travail. Au moment de son départ, il était instituteur et militant syndicaliste dans cette

6 L'Istiqlal, "parti de I'Indépendance ", a été créé pendant la colonisation en 1943. Après l'indépendance (en 1960), une scission a donné lieu à la création, par Mehdi Ben Berka, du parti socialiste marocain, I'Union Socialiste des Forces Populaires. Dans les années 1960 et 1970, ce dernier a joué un rôle important dans I'envoi des étudiants en URSS. 
organisation. De 1959 à 1961, il a occupé le poste de secrétaire de la jeunesse ouvrière, section de la ville de Safi. Pendant son séjour d'étude à Leningrad, il fut président de la section de I'Union Nationale des Étudiants du Maroc:

"L'Union Nationale des Étudiants du Maroc était très active en URSS. Dans chaque ville où il $y$ avait des étudiants marocains, il $y$ avait une section, sachant que le siège principal était implanté à Moscou. À Leningrad, c'est moi qui étais le chef de section. J'ai occupé ce poste pendant une année et nous étions très actifs. Par exemple, après l'enlèvement de Mehdi Ben Barka, nous avons protesté en organisant un setting face à l'ambassade du Maroc à Moscou et nous avons également envoyé des lettres de protestation au Palais Royal et au ministère de I'Intérieur " (Abderrahim El Attaoui).

Mais il convient de ne pas perdre de vue que hormis une poignée d'activistes, la plupart des étudiants qui partaient vers I'URSS en période d'agitations politiques, et au moment même où le régime de Hassan II " décapitait " I'opposition, étaient plus intéressés par les études et la préparation de leur avenir professionnel que par l'activité politique partisane ou syndicale. À leur retour au Maroc, la plupart de ces premiers diplômés d'URSS étaient beaucoup plus préoccupés par la lutte pour la reconnaissance de leur diplôme que par les batailles politiques du Maroc durant les " années de plomb " ${ }^{7}$. Les autorités marocaines développaient une certaine méfiance à l'égard des étudiants formés en URSS. Les diplômés de la période soviétique que nous avons interviewés rapportent qu'à leur retour au Maroc, ils étaient surveillés par la police marocaine :

"Lorsque j'ai postulé pour un poste d'architecte aux services de l'urbanisme du ministère de l'Intérieur en 1969, j'étais tout le temps surveillé discrètement par les renseignements. Ce n'est qu'après mon intégration qu'un collègue m'a dit que j'ai eu ce poste, car les renseignements n'ont rien trouvé. En fait, la plupart des diplômés de I'URSS des années 1960 et 1970 ont été surveillés par les services secrets. L'État a mis du temps pour comprendre que les étudiants diplômés de I'URSS, y compris ceux qui sont partis par le biais des partis, étaient des étudiants ordinaires et non pas des opposants au régime " (architecte diplômé de l'Institut d'Architecture de Moscou en 1968).

Les premières promotions d'étudiants parties se former en URSS au début des années 1960, dans un contexte politique national et international mouvementé, ont ouvert un nouvel espace migratoire pour les lycéens marocains. La voie vers les instituts et les universités soviétiques s'est ainsi progressivement consolidée contribuant à la diversification des mobilités internationales des étudiants marocains.

\section{Départs des étudiants marocains vers les instituts et les universités soviétiques dans les années 1970 et 1980}

Pour donner une idée du nombre d'étudiants marocains qui sont partis en URSS, particulièrement durant les années antérieures à la Perestroïka, nous nous sommes appuyés sur les données rapportées par Béthune et Leclerc-Olive

7 Les " années de plomb " est l'expression utilisée par les acteurs politiques et les médias au Maroc pour désigner les violences, les répressions, les tortures et les enlèvements qui ont marqué le règne d'Hassan II durant les années 1960-1970 et 1980. 
$(2013)^{8}$. Entre 1959 et 1979, 2605 étudiants marocains ont été inscrits dans les universités et instituts soviétiques. Une nette augmentation des départs a été enregistrée dans les années 1980. On est passé d'une moyenne de soixante-sept étudiants partant chaque année, entre 1956 et 1982, à une moyenne de quatrevingt étudiants entre 1983 et 1987. Ces mobilités, aussi anciennes que celles vers la France, ont joué un rôle important dans la formation d'une fraction importante des cadres et diplômés marocains. Cependant, à l'inverse de la mobilité vers la France, celle vers I'URSS n'a été favorisée ni par I'héritage colonial, ni par la proximité linguistique, géographique ou politique et encore moins par la présence d'une communauté de migrants marocains. C'est la conjugaison de plusieurs facteurs qui l'ont rendu possible : offre de bourses avec prise en charge complète par I'Union Soviétique, signatures d'accords de coopération permettant aux étudiants de bénéficier de bourses marocaines et soviétiques, prestige scolaire et social au Maroc des études proposées en URSS (médecine, ingénierie, physique, biologie, architecture, etc.), attractivité du positionnement technologique et politique de I'URSS pendant la Guerre froide, efficacité des relais institutionnels soviétiques, notamment le Centre Culturel Russe créé à Rabat en 1968. Dans les années 1970 et 1980, ce centre a joué un rôle non négligeable dans la diffusion d'une image positive des études en URSS auprès de la jeunesse marocaine étudiante. La filière d'étude Maroc-URSS a également été maintenue grâce à l'action des premières générations de diplômés des instituts et des universités soviétiques qui se sont organisées en association en 1977 avec la création à Rabat de l'AMLUIS (Association Marocaine des Lauréats des Universités et Instituts Soviétiques). Cette association, encore active aujourd'hui, a été pour des lauréats et des étudiants un organisme mobilisateur pour la reconnaissance des diplômes, un lieu de sociabilité pour les anciens lauréats et enfin un relais facilitant les départs des étudiants. Mais de tous les facteurs qui ont pesé sur les départs, le plus décisif concerne l'offre de bourses soviétiques. Elle a permis à plusieurs générations d'étudiants de poursuivre des études supérieures, de partir se former gratuitement et même de reconstruire des projets scolaires et des projets de vie dans des conditions idéales, après notamment des échecs au Maroc. Tel fut le cas d'Abderrahim qui, partit en 1961 avec un brevet obtenu au Maroc en 1957, est devenu un des premiers diplômés marocains d'URSS. Son parcours illustre celui de plusieurs étudiants marocains qui sans même avoir le baccalauréat ont fait des études supérieures :

" J'ai toujours voulu continuer mes études. Mais je n'avais pas le bac. Là-bas, ce n'était pas obligatoire pour entrer à l'université. J'ai passé une année préparatoire avant d'être orienté vers la faculté de philologie de l'université de Leningrad. J'ai passé avec succès les tests de fin d'année et par la suite, les autorités soviétiques nous ont donné un papier attestant que nous disposons du niveau nécessaire pour accéder à l'université. II faut aussi dire qu'il y a eu d'autres facteurs qui ont attiré les étudiants vers I'URSS à mon époque. Bien sûr, il y avait la bourse, la disponibilité de logement et les vêtements chauds pour passer I'hiver. Mais il y a eu surtout le fait qu'on pouvait accéder à l'enseignement supérieur sans bac et il y avait également des étudiants qui sont partis là-bas par admiration du système

8 Les auteurs présentent des données statistiques brutes sur l'accueil des étudiants étrangers en URSS depuis la fin des années 1950. Ils se sont appuyés sur plusieurs sources : les données de la CIA, les données de I'UNESCO et les données collectées par Katsakioris (dans ce dossier thématique) auprès du ministère de l'Éducation à Moscou. 
socialiste " (Abderrahim, diplômé en 1968 de la faculté de philologie de l'université de Leningrad et enseignant-chercheur retraité de la faculté des lettres de Rabat).

Tous les diplômés que nous avons interviewés ont eu accès à une bourse qui a déclenché le projet d'étude en URSS. Le système soviétique de bourse était à la fois souple et attractif. Le montant attribué aux Marocains, et plus généralement aux étudiants étrangers, était supérieur à celui octroyé aux nationaux et proche du revenu d'un fonctionnaire diplômé de l'État soviétique :

"Nous étions vraiment bien là-bas, on avait une bourse mensuelle de quatre-vingt-dix roubles. On avait également un billet d'avion pour arriver et repartir une fois les études terminées. Pour vous donner une idée de l'importance de notre bourse à cette époque, on avait une bourse de quatre-vingt-dix roubles et un médecin ou un ingénieur de l'État soviétique avait un salaire de 100 roubles " (Omar, architecte diplômé de l'Institut d'Architecture de Moscou en 1968).

En plus du montant intéressant de la bourse, les critères de son attribution n'étaient pas sévères. Mais la demande des étudiants marocains était plus importante que I'offre. II y avait bien sûr des critères méritocratiques, mais le réseau de relations fut souvent décisif pour accéder à ces bourses qui étaient octroyées aussi bien par le ministère de l'Enseignement supérieur et de la Formation des cadres, que par les partis politiques, les syndicats et les associations (I'Union Marocaine du Travail, I'Union Nationale des Étudiants du Maroc, I'Association de l'Amitié Marocaine-Soviétique, l'Association Marocaine des Lauréats des Universités et Instituts Soviétiques, I'Union Marocaine d'Action Féminine, etc.). Tous ces organismes politiques et associatifs ont distribué les bourses soviétiques plutôt selon une logique clientéliste. Les militants et les connaissances ont été privilégiés. Le récit de Mohammed, aujourd'hui pharmacien d'officine, parti en 1988 est révélateur à ce propos :

"Mon frère était à l'époque membre de Chabiba Al Itihadia lorganisme de jeunesse relevant de I'Union Socialiste des Forces Populaires) et c'est grâce à son aide que j'ai pu avoir une bourse de I'URSS. J'avais aussi une bourse marocaine. Je suis parti en 1988. La même année, j'ai eu une inscription en France à Nancy pour suivre des études d'ingénieur en génie civil. Je suis effectivement parti à Nancy même sans bourse. Mais quand mon frère m'a informé que j'avais la bourse de I'URSS, je suis rentré au Maroc immédiatement pour me préparer afin de partir là-bas étudier la pharmacie "

(Mohammed, pharmacien parti en 1988).

Si les bourses distribuées par les partis politiques, les syndicats, les associations et même par l'État marocain n'étaient pas forcément des bourses d'excellence, elles ont, en revanche, permis à des étudiants d'origine modeste, faiblement dotés sur le plan scolaire et social, de poursuivre leurs études dans des filières très sélectives au Maroc. Les diplômés d'URSS que nous avons interviewés étaient plutôt d'origine modeste et leur parcours scolaire ne fut pas un parcours d'excellence. Ils sont fils d'ouvriers, de petits commerçants ou d'artisans. Certains, dont le père était décédé, étaient financés par le grand frère, lui-même petit fonctionnaire. Ils ont eu le baccalauréat le plus souvent avec mention " assez bien ", et les notes obtenues ne leur permettaient pas d'accéder aux grandes écoles marocaines, encore moins aux écoles françaises. Mais parmi les diplômés d'URSS, il n'y avait pas que des étudiants issus des milieux popu- 
laires. Ainsi les classes moyennes urbaines ont trouvé dans les études en URSS (et aujourd'hui en Russie) une opportunité pour scolariser leurs enfants dans des filières garantissant une bonne rentabilité du diplôme. Parmi les diplômés d'URSS, on compte également des fils de parlementaires, d'instituteurs, de fonctionnaires et de cadres. Cependant, les étudiants marocains les plus dotés en capitaux (scolaire, social, familial), destinés à occuper une place privilégiée parmi les élites dirigeantes du pays, partent plutôt en France pour suivre leurs études dans les filières d'excellence (Vermeren, 2011 et 2002 ; Scarfò, 2004).

\section{De I'URSS à la Russie : les transformations de la période post soviétique (1991-2015)}

Après la dislocation de I'URSS en 1991 et le passage à l'ère post-soviétique, les conditions d'accès aux études en Russie et dans les autres pays de l'Est ont connu de profondes modifications : diminution des bourses d'État, augmentation des inscriptions payantes dans des instituts privés, mais aussi dans les anciennes universités publiques, apparition d'agences privées d'aide pour organiser la migration, orientation vers les études médicales et pharmaceutiques au détriment des sciences de l'ingénieur.

\section{L'attractivité de la Russie après 1991}

Dans les années 1990 et 2000, les mobilités internationales des étudiants marocains se sont diversifiées, même si la France demeure le principal pays d'accueil (Marchandise, 2013 : 131). Les pays de l'Est de I'Europe (la Russie, I'Ukraine, la Roumanie, etc.) ont contribué à cette diversification en accueillant de plus en plus d'étudiants marocains.

Tableau 1 : Étudiants marocains inscrits à l'étranger dans les principaux pays d'accueil (en effectif)

\begin{tabular}{|c|c|c|c|c|c|c|c|c|c|c|c|c|c|c|c|}
\hline & 1999 & 2000 & 2001 & 2002 & 2003 & 2004 & 2005 & 2006 & 2007 & 2008 & 2009 & 2010 & 2011 & 2012 & 2013 \\
\hline France & 18849 & 21048 & 24284 & 29504 & 34826 & 32802 & 29859 & 29299 & 27684 & 26998 & 27051 & 27467 & 28463 & 28778 & 23617 \\
\hline Allemagne & 5416 & 5882 & 6285 & 6960 & 7616 & 8305 & 8227 & 4784 & 4369 & 3699 & 3748 & 3306 & - & - & 2298 \\
\hline Belgique & 4527 & 5355 & 4894 & 5146 & 2658 & 52 & 68 & 55 & 59 & 62 & 179 & 205 & 228 & 197 & 841 \\
\hline Espagne & 2258 & 3144 & 2877 & 3263 & 3468 & 1450 & 1517 & 1613 & 1782 & 2735 & 3165 & 3514 & 3117 & 3209 & 3071 \\
\hline Italie & 235 & 244 & 332 & 361 & 518 & 664 & 776 & 813 & 1017 & 1207 & 1398 & 1554 & 1715 & 1766 & 1583 \\
\hline Pays-Bas & 1829 & 1874 & 1956 & 1953 & 1664 & 429 & 376 & 297 & 234 & 246 & 193 & 156 & 133 & 122 & - \\
\hline USA & 1306 & 1484 & 1662 & 2102 & 2034 & 1835 & 1641 & 1555 & 1229 & 1133 & 1150 & 1110 & 1178 & 1264 & 1237 \\
\hline Canada & 765 & 768 & 795 & 714 & 729 & 777 & 816 & 2652 & 1587 & 1770 & 1440 & 1344 & 1311 & 1185 & - \\
\hline Roumanie & 300 & 286 & 235 & 216 & 175 & 130 & 109 & 89 & 117 & 201 & 258 & 351 & 408 & - & 606 \\
\hline Ukraine & - & - & - & - & - & - & 679 & 632 & 671 & 799 & 833 & 851 & 871 & 1022 & 1189 \\
\hline Russie & - & - & - & - & - & - & - & - & - & 867 & 860 & - & 886 & - & - \\
\hline
\end{tabular}

- Pas de données.

Source : Statistiques de I'UNESCO. 
Les données statistiques de I'UNESCO utilisées dans le tableau 1 ne permettent pas d'avoir des chiffres pour la totalité des pays d'accueil des étudiants marocains et pour l'ensemble des années. Elles offrent par contre la possibilité de donner des éléments de compréhension sur les évolutions de la mobilité étudiante selon les principaux pays d'accueil. Il ressort que la migration étudiante marocaine est traversée par plusieurs courants. Nous nous intéressons particulièrement ici au courant des pays de l'Est de l'Europe, notamment à la Russie. Ce pays forme un courant marginal comparé à celui de la France et dans une moindre mesure de l'Allemagne et de l'Espagne. Cependant, l'effectif des étudiants marocains inscrits en Russie augmente sensiblement au cours des années 2000 (Tableau 2). En 2013, I'Ukraine est le premier pays de l'Europe de l'Est à accueillir des étudiants marocains, suivi par la Fédération de Russie.

\section{Tableau 2 : Étudiants marocains inscrits en Ex URSS-Russie}

\begin{tabular}{cc}
\hline Année & Eff. \\
\hline 1988 & 650 \\
2008 & 867 \\
2011 & 886 \\
\hline
\end{tabular}

Source : UNESCO ; Béthune et Leclerc-Olive (2013).

L'AMLUIS estime qu'entre 1960 et 2015, le nombre des cadres marocains formés en Russie atteint environ 18000 diplômés, dont 3500 formés avant 1991 et 14500 après la dislocation de I'URSS. L'engouement des étudiants marocains pour les études en Russie ne se dément donc pas. Les raisons en sont multiples et variées. Elles ne sont probablement pas à rechercher uniquement dans les facilités d'obtention du visa ou dans le coût abordable des études. Elles sont également en lien avec le renforcement de l'attractivité internationale de I'enseignement supérieur en Russie (Kastouéva-Jean, 2009) et la forte demande sociale au Maroc pour les études à l'étranger (Gérard, 2008 ; Marchandise, 2013 ; Balac, 2010). La mobilité internationale jouit d'un prestige important au sein du système national d'enseignement supérieur marocain au point que la formation à l'étranger s'est instituée au Maroc comme un prolongement du système national de formation (Dubois, Chemkhi et lbourk, 2012) ${ }^{9}$. Accéder à un diplôme étranger étant réputé multiplier les possibilités de réussite professionnelle, la mobilité est au centre des stratégies éducatives des familles marocaines. Dans un contexte de massification de l'enseignement supérieur, de renforcement du caractère sélectif des filières scolaires locales d'excellence (ingénierie, médecine, pharmacie, etc.) et de durcissement des conditions d'accès aux études en France, la Russie s'est imposée comme une destination offrant aux étudiants marocains une deuxième chance. Le départ pour la formation supérieure dans ce pays a permis aux étudiants marocains de contourner le caractère sélectif des études supérieures au Maroc et d'échapper au chômage de masse qui frappe les diplômés des universités marocaines à accès ouvert (Mellakh,

9 Selon l'enquête menée par les auteurs auprès des étudiants de l'université de Marrakech, $66 \%$ des étudiants souhaitent poursuivre leurs études à l'étranger et $34 \%$ ont effectivement commencé à entreprendre les démarches nécessaires pour concrétiser leurs projets de départ. 
2000). Interrogés sur les circonstances de leur départ, un trait commun apparaît dans les réponses des interviewés, au-delà de la singularité de leur parcours et de la diversité des contextes qui ont amené à la mobilité. Les diplômés de l'ancienne Union Soviétique et de l'actuelle Fédération de Russie ont en commun de présenter leur départ comme une opportunité qu'il fallait saisir pour un projet d'étude. L'idée du départ s'est alors concrétisée très vite et sans longue préparation préalable. Partir se former en Russie n'a pas été forcément le premier choix, mais ils étaient, en fin de compte, tous satisfaits de la Russie comme destination. Ce fut le cas, aussi bien de Mahdi, médecin dentiste diplômé en 2015 de l'université IP Pavlov de la ville de Ryzain, que de Youssef, pharmacien diplômé de I'université de Saint-Pétersbourg en 1999.

Pour Mahdi, les notes obtenues au baccalauréat ne permettent nullement d'espérer une inscription dans une grande école marocaine et ses moyens financiers sont insuffisants pour satisfaire son premier vœu d'entreprendre des études de gestion et de commerce au Canada :

"Après le bac, je n'avais pas un projet précis. Je ne savais pas exactement quoi faire. Au début, j'ai voulu faire commerce et gestion à l'université de Sherbrooke au Canada. Mais après une longue discussion avec mon père, j'ai opté pour la médecine dentaire en Russie. C'était plus facile, car les études au Canada étaient plus chères " (Mahdi).

Quant à Youssef, son départ à Saint-Pétersbourg pour devenir pharmacien a eu lieu après le refus de son visa pour la France. Il a réussi à décrocher une préinscription en pharmacie à l'université de Bordeaux. Mais il n'a pas pu partir :

"Au début, j'ai voulu faire pharmacie en France. J'ai passé avec succès les différentes phases de sélection de campus France et j'ai bien eu une inscription à Bordeaux. Mais je n'ai pas pu y aller faute d'obtention de visa. Je me suis inscrit à HEM -Casa (École supérieure privée de Management) pour ne pas perdre l'année. Mais je n'ai pas continué.

En fait, je n'ai jamais pensé faire des études en Russie. Tout est parti grâce à un ami de

lycée qui est parti là-bas avant moi. II m'a parlé de la possibilité d'y aller et comme j'ai toujours voulu faire pharmacie, je n'ai pas hésité. Je suis allé vraiment par hasard. Je n'ai jamais pensé que j'allais partir en Russie, car au début j'ai voulu partir en France comme je t’ai déjà dit. C'est cet ami de lycée qui était déjà inscrit là-bas qui m'a ramené une inscription et m'a montré toutes les démarches à suivre "(Youssef).

II serait cependant réducteur de considérer la Russie uniquement comme une simple alternative ou un choix par défaut. Avec une tradition de départ qui remonte aux années 1960, ce pays n'est plus une destination inconnue chez les étudiants marocains. Dans un contexte de durcissement des politiques migratoires, la Russie est devenue un choix pragmatique. Les étudiants désireux de poursuivre leurs études à l'étranger se sont adaptés à la nouvelle donne migratoire : forte sélectivité des destinations habituelles à l'Ouest, et ouverture de nouvelles possibilités migratoires à l'Est. D'une part, la France a instauré un contrôle des études avant même le départ, à travers le dispositif Campus France (Spire, 2009) et d'autre part des pays comme la Russie et I'Ukraine ont renforcé leur attractivité bien que les études soient à présent payantes. 


\section{L'augmentation des formations payantes après 1990}

Après la dislocation de I'URSS, la Fédération de Russie a développé, au sein même des établissements publics, des activités commerciales pour faire face aux exigences de l'auto-financement de ses établissements universitaires. Elle a mis en place une politique " d'accueil d'étudiants qui peuvent payer leurs études (les kontraktniki), par opposition aux bjudzetniki dont la scolarité est prise en charge par l'établissement "(Groppo, 2009 : 146). C'est l'irruption après 1991 des formations payantes au sein même des universités d'État qui a attiré les étudiants marocains et leurs familles, au point que tout un marché local d'envoi des étudiants vers la Russie et les autres pays de l'Est (I'Ukraine, la Roumanie, etc.) s'est développé au Maroc pour répondre à une demande sociale croissante. Ce marché dispose de ses propres agents et courtiers qui servent de relais pour faciliter la migration. D'anciens lauréats se sont transformés en intermédiaires. Des cabinets privés de formation et de gestion des ressources humaines proposent également des prestations pour aider les étudiants à obtenir des inscriptions. Le RACUS (Centre Russo-arabe des Services Universitaires) ${ }^{10}$ a également joué un rôle important dans l'accès aux études payantes en Russie. Ces nouveaux acteurs se sont ajoutés aux anciennes structures - AMLUIS et le Centre Culturel Russe pour l'Éducation et la Science de Rabat - qui ont depuis longtemps contribué à la promotion des études en Russie auprès de la jeunesse marocaine scolarisée. Le caractère persistant de la mobilité des étudiants marocains vers la Russie tient à l'intervention de ces différents acteurs. Aujourd'hui, un des principaux leviers de cette mobilité est l'auto-financement par les familles. Les personnes interviewées parties par la voie privée ont systématiquement souligné le rôle déterminant des intermédiaires et du financement privé par la famille dans l'aboutissement de leur projet de départ :

"La première fois, je suis allé au RACUS avec mes parents pour avoir les informations afin d'instruire le dossier. En fait, ce n'était pas très compliqué. II n'y avait pas de conditions draconiennes. Il y avait surtout des conditions financières. Nous avons pu organiser le voyage en quelques jours " (Mehdi, médecin dentiste diplômé en 2015 de l'université IP Pavlov de la ville de Ryzain).

"C'est mon père qui a financé mes études là-bas. Chaque année, je payais des frais d'inscription. Mon père m'envoyait mensuellement 200 dollars pour vivre. Maintenant, les études en Russie sont plus chères comparées à mon époque " (Youssef, pharmacien diplômé de l'université de Saint-Pétersbourg en 1999).

Il est important de souligner qu'avec l'augmentation des formations payantes, la mobilité vers l'Est est devenue plus favorable aux étudiants issus de familles qui peuvent supporter le coût de plus en plus onéreux des études. Le recours à la famille pour construire un projet de formation dans un pays de l'Est prend aujourd'hui beaucoup plus d'importance. Les possibilités de départ se sont fortement réduites pour les étudiants qui ne peuvent pas bénéficier d'un soutien

10 Le RACUS est une agence créée pour promouvoir I'offre éducative russe à l'étranger. Elle a vu le jour en 1991 à Saint-Pétersbourg suite à une loi autorisant les universités d'État de la Fédération de Russie à inscrire les étudiants étrangers moyennant le payement des frais d'études. 
financier durant leurs études. Le passage de I'URSS à la Russie s'est ainsi traduit par un déclassement des étudiants qui à l'époque de I'URSS pouvaient bénéficier des bourses soviétiques pour réaliser leur projet de mobilité. Bien que le coût des études dans les pays de l'Est ait augmenté, étudier en Russie ou en Ukraine reste pourtant moins cher qu'étudier en France. Ces pays attirent donc une population étudiante économiquement différente de celle qui s'oriente vers la France. Si, dans l'ensemble, les diplômés des pays de l'Est d'après 1991 sont issus de milieux sociaux variés (classes moyennes, milieux modestes et milieux aisés), ils sont toutefois plus souvent d'origine urbaine et ils sont issus de familles dont le père, ou un proche sont en mesure de financer le départ.

\section{L'orientation vers les études pharmaceutiques}

Après 1991, ce sont principalement les formations permettant d'exercer des professions libérales, valorisées scolairement et socialement au Maroc, qui ont attiré les étudiants à destination de la Russie. Selon une note de l'ambassade du Maroc à Moscou, " environ 3500 étudiants marocains poursuivent en 2013-2014 leurs études en Russie dans des disciplines médicales et paramédicales ". Et une des évolutions les plus marquantes de cette mobilité étudiante marocaine vers la Russie est l'augmentation des choix en faveur des disciplines médicales et paramédicales et notamment pour la pharmacie. Cette formation est massivement privilégiée parce qu'elle permet d'une part une insertion professionnelle rapide après le retour au Maroc et d'autre elle correspond au marché de l'emploi où dominent les emplois privés - alors que jusqu'en 1990 les emplois publics étaient les plus nombreux. Mais les départs spontanés et massifs des étudiants marocains au cours des années 1990 et 2000 pour se former en pharmacie en Russie ou dans d'autres pays d'Europe de l'Est ont donné lieu à un fait inattendu : la massification rapide de la profession et une forte tension sociale autour de la reconnaissance du diplôme (Mellakh, 2015).

Tableau 3 : Pharmaciens marocains selon les principaux pays d'obtention de diplôme (en effectif)

\begin{tabular}{lcc}
\hline & 2001 & 2012 \\
\hline Maroc & 797 & 1382 \\
France & 1821 & 1843 \\
Belgique & 512 & 346 \\
Espagne & 341 & 1278 \\
Ex URSS/Russie & 1593 & 3917 \\
Ukraine & 270 & 346 \\
Roumanie & 228 & 691 \\
Sénégal & 180 & 230 \\
Algérie & 176 & 220 \\
Tunisie & 341 & 461 \\
\hline
\end{tabular}

Source : Ordre National des Pharmaciens du Maroc. 
Tableau 4 : Pharmaciens marocains selon les lieux d'obtention de diplôme (2012)

\begin{tabular}{lcc}
\hline & Eff. & $\%$ \\
\hline Maroc & 1382 & 12 \\
Maghreb & 690 & 6 \\
Europe de l'Est & 4952 & 43 \\
Europe de l'Ouest & 3455 & 30 \\
Afrique subsaharienne & 235 & 2 \\
Autres & 806 & 7 \\
Total & $\mathbf{1 1 5 2 0}$ & $\mathbf{1 0 0}$ \\
\hline
\end{tabular}

Source : Ordre National des Pharmaciens du Maroc.

Parmi les 11520 pharmaciens d'officine inscrits en 2012 dans le tableau de I'Ordre, 3917 ont été formés en Russie contre seulement 1382 formés au Maroc et 1843 en France. En 2012, les pharmaciens diplômés des pays de l'Est (Russie, Ukraine, Roumanie, Bulgarie, etc.) représentent $43 \%$ de l'ensemble des pharmaciens en activité au Maroc (34 \% ont été diplômés en Russie). Leur effectif a plus que doublé entre 2001 et 2012 (cf. Tableau 3).

Un déplacement important s'est opéré au niveau des lieux de formation à l'étranger des pharmaciens marocains au cours des décennies 1990 et 2000. Avant les années 1990, les étudiants marocains en quête d'une formation en pharmacie avaient plutôt l'habitude de se diriger vers les anciens foyers de la migration marocaine de travail, notamment la France, et dans une moindre mesure en Belgique. L'ouverture des pays de l'Est après 1991 leur a offert une nouvelle opportunité. Elle s'est traduite par une augmentation rapide du nombre de pharmaciens marocains diplômés en Russie, en Ukraine et en Roumanie au cours des années 2000 ; cet afflux massif d'étudiants s'explique par la forte sélectivité des études médicales et pharmaceutiques au Maroc et en France. Alors qu'on note une augmentation du nombre de jeunes Marocains accédant à l'enseignement supérieur, les études médicales et pharmaceutiques ont renforcé leur caractère méritocratique et sélectif (Mellakh, 2000). La candidature au concours d'accès en première année de pharmacie n'est autorisée que pour les étudiants titulaires d'un DEUG en biologie (diplôme obtenu après deux années d'études dans les facultés des sciences). Ce système d'admission exclut les bacheliers de l'accès direct au concours. D'autres mesures ont également contribué à rendre très difficile l'accès aux études pharmaceutiques au Maroc même. La faculté de médecine et de pharmacie de Rabat a été longtemps la seule faculté du pays à dispenser cette formation, et elle n'est ouverte que depuis 1986. Et ce n'est qu'en 2010 qu'une nouvelle section pharmaceutique a été créée au sein de la faculté de médecine de Casablanca. Les nouvelles facultés de médecine et de pharmacie créées à Fès et à Marrakech en 1999 se sont limitées à l'enseignement de la médecine. Ce contexte encourage les parents d'élèves, à la recherche d'un bon placement scolaire et social pour leurs enfants, à s'orienter vers la filière russe de formation en pharmacie. Cette filière payante donne aussi une seconde chance aux enfants des classes moyennes qui ont accédé à l'enseignement supérieur, mais qui ne peuvent pas décrocher facilement une inscription dans les facultés de médecine et de pharmacie marocaines ou françaises. 


\section{Reconnaissance sociale des diplômes obtenus à l'Est}

La formation dans les pays de l'Est de l'Europe a ouvert à plusieurs générations d'étudiants marocains la possibilité d'obtenir un diplôme supérieur, d'internationaliser leur formation et de s'insérer sur le plan professionnel au Maroc. Mais a-t-elle fourni à ces étudiants une porte d'accès à l'élite dirigeante du pays, jusqu'ici dominée par les diplômés des filières françaises d'excellence? Cette question pose directement celle de la mobilité étudiante comme facteur de production ou de reproduction des élites marocaines déjà traitée par divers auteurs (Vermeren, 2011 ; Scarfò, 2004). Répondre à cette question requiert de disposer d'éléments pour retracer les trajectoires scolaires et professionnelles des diplômés de l'Est en fonction de leur origine sociale, du pays de formation, des filières d'étude et du rythme de l'évolution de leur carrière. Nos enquêtes ne permettent pas de répondre de manière définitive à ces questions. Elles permettent néanmoins de formuler l'hypothèse que ces diplômés ont eu des carrières professionnelles valorisantes grâce à leur formation à l'Est de l'Europe, mais que la plupart d'entre eux ne fait pas partie des élites dirigeantes du pays. Si la formation à l'Est a permis de déclencher un processus de réussite scolaire et $d$ 'insertion professionnelle, elle ne permet pas d'augmenter, d'une manière substantielle, les chances d'accès à l'élite dirigeante du pays, comme c'est le cas pour les diplômés marocains des grandes écoles françaises. Dans la lutte pour I'accès aux postes de pouvoir, les lauréats des pays de l'Est ont été fortement fragilisés par la perception négative de leurs études à l'Est. Les diplômes obtenus en Europe de l'Est sont réputés être de mauvaise qualité et faciles à acquérir. À leur retour au Maroc, les diplômés ne jouissaient pas d'une pleine reconnaissance de la part des professions bien établies au Maroc (médecins, pharmaciens, ingénieurs, etc.) ; en conséquence de quoi, ils sont entrés en confrontation directe avec les élites professionnelles sur place. Le cas des médecins et des pharmaciens est significatif à cet égard. Les pharmaciens, partis en grand nombre étudier en Russie et en Ukraine dans des filières payantes après 1991, ont été confrontés à leur retour aux difficultés d'insertion dans une profession dominée jusqu'alors par les diplômés formés en France. Les diplômes obtenus à l'Est ont été contestés par la profession. Au début des années 2000, l'Ordre National des Pharmaciens du Maroc a exigé que les diplômés de l'Est soient systématiquement soumis à un contrôle de validation des connaissances, ce qui fut à l'origine d'un large mouvement de protestation des nouveaux lauréats en provenance des pays de l'Est (Mellakh, 2015). Les rumeurs véhiculées par la presse marocaine de l'époque sur la falsification et l'achat des titres scolaires après l'effondrement de I'URSS n'ont pas manqué de délégitimer les diplômes de l'Est et de stigmatiser leurs détenteurs. Les médecins, les pharmaciens et les architectes que nous avons interviewés sont aujourd'hui bien insérés dans leur profession. Mais ils ont tous été confrontés à ce problème épineux de la reconnaissance du diplôme par le ministère de l'Enseignement supérieur ou par les professions qu'ils voulaient intégrer. Le déni du diplôme est au centre de l'expérience du retour vécue par les différentes générations de lauréats des pays de l'Est, comme le montre les récits d'Omar, architecte diplômé de I'Institut d'Architecture de Moscou en 1968, d'Abderrahim, enseignant-chercheur retraité de la faculté des lettres de Rabat, diplômé de I'université de Leningrad en 1967 et de Mahdi, médecin dentiste, diplômé en 2015 de l'université IP Pavlov de la ville de Ryzain : 
"Personnellement, j'étais également confronté à ce problème d'équivalence de diplôme. Pourtant, je suis diplômé de l'Institut d'Architecture de Moscou qui a aujourd'hui 250 ans d'histoire. II a été créé en 1749. J'ai étudié dans cet institut pendant 7 ans sans compter l'année préparatoire. Lorsque je suis entré en 1968, le ministère de l'Enseignement supérieur n'a pas reconnu immédiatement mon diplôme. II a adressé une demande de renseignement sur mon diplôme à l'ordre national des architectes de France et c'est uniquement après son avis favorable que j'ai eu l'équivalence " (Omar, architecte).

" Lorsque je suis rentré en 1967, j'ai déposé ma demande d'embauche à plusieurs ministères. Mais je n'ai reçu aucune réponse. J'ai passé une année à chercher du travail au moment où le pays n'avait pas beaucoup de cadres diplômés et ceci à cause de la méconnaissance des autorités, de mon diplôme de philologue obtenu en URSS. Ce

fut uniquement grâce à l'aide d'une personne qui connaissait très bien Mohammed EI Fassi, grand nationaliste et recteur de l'université Mohammed V de l'époque, que j'ai pu décrocher un rendez-vous pour un entretien d'embauche à la faculté des lettres de Rabat.

J'ai réussi l'entretien. Mais il a fallu régler le problème de l'équivalence. Mon diplôme de docteur de la faculté de philologie de l'université de Leningrad n'a pas été reconnu en tant que tel. Ils m'ont donné l'équivalence et le salaire d'une licence pour occuper un

poste d'enseignant à la faculté et évidemment j'ai protesté contre cela. Pour régler le problème, ils m'ont demandé de traduire ma thèse en français, puis ils l'ont envoyée à un jury de la faculté des lettres de Rabat pour l'évaluer. C'est seulement après leur rapport d'évaluation positif que j'ai eu le grade de docteur à l'université Mohammed V de Rabat "

(Abderrahim, enseignant-chercheur).

"La procédure qu'ils nous ont imposé pour obtenir l'équivalence est compliquée et injuste. Ça nous fait perdre bêtement une année. Quand tu rentres, tu as l'impression que ton diplôme ne sert à rien. II faut attendre l'équivalence. Pour l'obtenir, il faut commencer les démarches là-bas : j'ai demandé la liste des notes, des matières et des heures enseignées pendant toute la durée de la formation. J'ai traduit le tout en français et j'ai certifié le diplôme et tous les autres documents obtenus là-bas à l'ambassade du Maroc. Par la

suite, j'ai déposé mon dossier d'équivalence au ministère de l'Enseignement supérieur. Mon dossier a été examiné par une commission. J'ai eu la réponse positive de la commission. Ils m'ont envoyé, comme il est d'usage, une convocation pour prendre contact avec

le doyen de la faculté de médecine et de pharmacie de Rabat afin de suivre les cours supplémentaires et organiser mon stage à l'hôpital, nécessaire pour avoir l'équivalence "

(Mahdi, médecin dentiste).

\section{Conclusion}

Malgré les difficultés rencontrées pour valoriser le diplôme en début de carrière, les étudiants marocains partis se former dans les pays de l'Est, ont tiré des bénéfices scolaires et sociaux de leurs expériences migratoires. Ils n'accèdent pas facilement aux postes clefs au sommet de l'État ou des entreprises marocaines, mais ils parviennent à rentabiliser leurs diplômes d'une manière satisfaisante, et à trouver une place dans un marché de l'emploi de plus en plus concurrentiel.

La production de diplômés marocains par les pays d'Europe de l'Est est un phénomène ancien et paradoxalement peu connu. Notre étude, toujours en cours, ne prétend ni à l'exhaustivité, ni à une analyse définitive du phénomène. 
II s'agit uniquement d'apporter un premier éclairage sur le contexte marocain qui a favorisé cette forme de mobilité étudiante assez particulière. II reste à étudier en profondeur les différentes facettes de l'expérience migratoire de ces étudiants et les liens entre l'évolution du contexte géopolitique et ces recompositions des mobilités internationales des étudiants marocains.

\section{Références bibliographiques}

Balac Ronan (2010) Les mobilités internationales des étudiants marocains, Espace, Populations, Sociétés, 2-3, pp. 395-411.

Balac Ronan (2008) Les étudiants marocains expatriés en France, 1970-2005: une migration en mutation rapide, in Étienne Gérard Éd., Mobilités étudiantes Sud-Nord. Trajectoires scolaires de Marocains en France et insertion professionnelle au Maroc, Paris, Publisud, pp. 29-59.

Berramdane Abdelkhaleq (1987) Le Maroc et l'Occident (1800-1974), Paris, Karthala, 448 p.

Béthune Jean-Pierre et Leclerc-Olive Michèle (2013) Quelques données statistiques sur les flux d'étudiants internationaux. Commentaires et questions, [en ligne]. URL : http://riae.hypotheses.org/category/colloques/mohammedia-2013

Bianquis Arielle (2000) Une évolution contrastée. L'exemple de l'enseignement supérieur dans la Fédération de Russie, Revue internationale d'éducation de Sèvres, 28, pp. 79-89.

Dubois Thomas, Chemkhi Amine et lbourk Aomar (2012) L'intention de mobilité internationale des étudiants de l'université de Marrakech, Maghreb-Machrek, 2011, pp. 55-67.

Garneau Stéphanie (2008) L'émigration marocaine au Canada : contextes de départ et diversité des parcours migratoires, Diversité urbaine, 8 (2), pp. 163-190.

Geisser Vincent (Éd.) (2000) Diplômés maghrébins d'ici et d'ailleurs, trajectoires sociales et itinéraires migratoires, Paris, CNRS, $332 \mathrm{p}$.

Gérard Étienne (Éd.) (2008) Mobilités étudiantes Sud-Nord. Trajectoires scolaires de Marocains en France et insertion professionnelle au Maroc, Paris, Publisud, 379 p.

Groppo Armelle (2009) L'enseignement supérieur en Russie aujourd'hui, Revue Russe, 33, pp. 145-158, [en ligne]. URL : http//www.persee.fr/doc/russe_11610557_2009_num_33_1_2394

Kastoueva-Jean Tatiana (2009) Russie : la réforme de l'enseignement supérieur, Revue internationale d'éducation de Sèvres, 50, pp. 14-16.

Katsakioris Constantin (2007)Transferts Est-Sud. Échanges éducatifs et formation de cadres africains en Union soviétique pendant les années soixante, OutreMers, 354, pp. 83-106.

Laffort Bruno (2009) L'immigration des intellectuels marocains en France : regards sur une génération d'étudiants étrangers, Paris, Karthala, 439 p.

Marchandise Sabrina (2013) Territoires éphémères. Les réseaux sociaux des étudiants marocains en mobilité internationale, Thèse en géographie, Université Montpellier III-Paul Valéry, 451 p. 
Mazzella Sylvie (Éd.) (2009) La mondialisation étudiante. Le Maghreb entre Nord et Sud, Paris, Karthala-IRMC, $404 \mathrm{p}$

Mellakh Kamal (2015) La formation des pharmaciens marocains dans les pays de l'Est de l'Europe, in Monique de Saint-Martin, Scarfò Ghellab Grazia et Kamal Mellakh Éds., Étudier à I'Est. Expériences de diplômés africains, Paris, KhartalaFMSH, pp. 205-214.

Mellakh Kamal (2000) L'expansion scolaire et universitaire au Maroc : aspects et enjeux, in Vincent Geisser et Saïd Ben Sedrine Éds., Étudiants et diplômés maghrébins en devenir dans les sociétés en mutation : trajectoires d'insertion et itinéraires migratoires, Paris, CNRS, pp. 92-101.

Rhaouti Mohamed Laghdaf (2007) Le Code du médicament et de la pharmacie au Maroc [en arabe], Rabat, 234 p.

Saint-Martin Monique de, Scarfò Ghellab Grazia et Mellakh Kamal (Éds.) (2015) Étudier à l'Est. Expériences de diplômés africains, Paris, Khartala-FMSH, 298 p.

Scarfò Ghellab Grazia (2015) Les meilleures années de notre vie. Des ingénieurs marocains formés en URSS, in Monique de Saint-Martin, Scarfò Ghellab Grazia et Kamal Mellakh Éds., Étudier à l'Est. Expériences de diplômés africains, Paris, Khartala-FMSH, pp. 187-201.

Scarfò Ghellab Grazia (2004) Les écoles d'ingénieurs marocaines, lieu de passage des élites ? Le cas des élèves ingénieurs de l'École Hassania des Travaux Publics de Casablanca, in Éric Gobe Éd., L'ingénieur moderne au Maghreb (XIXe-XXe siècles), Paris, Maisonneuve \& Larose-IRMC, pp. 223-236.

Spire Alexis (2009) Contrôler et choisir. La sélection des étudiants candidats à l'émigration vers la France, in Sylvie Mazzella Éd., La mondialisation étudiante. Le Maghreb entre Nord et Sud, Paris, Karthala-IRMC, pp. 79-95.

Sy Harouna (2011) Marché de I'enseignement supérieur Sud-Sud. Les carabins marocains à I'université de Dakar, in Michèle Leclerc Olive, Grazia Scarfò Ghellab et Anne-Catherine Wagner Éds., Les mondes universitaires face aux logiques du marché : circulation des savoirs et pratiques des acteurs, Paris, Karthala, pp. 93-106.

Vermeren Pierre (2011) La formation des élites marocaines, miroir de la mondialisation ?, Le Télémaque, 39, pp. 53-66.

Vermeren Pierre (2002) École, élite et pouvoir au Maroc, Rabat, Édition des Alizés, $584 \mathrm{p}$

Yengo Patrice (2011) Jalon pour une historiographie des élites africaines formées dans le Bloc soviétịque, in Michèle Leclerc Olive, Grazia Scarfò Ghellab et AnneCatherine Wagner Éds., Les mondes universitaires face aux logiques du marché : circulation des savoirs et pratiques des acteurs, Paris, Karthala, pp. 135-148. 


\section{Kamal Mellakh}

\section{... La formation des étudiants marocains dans les pays de I'Est de I'Europe (1960-2015)}

Les pays de l'Europe de l'Est constituent une composante ancienne et assez particulière du système marocain de la formation à l'étranger. Depuis le début des années 1960, les étudiants marocains ont trouvé dans les systèmes universitaires des pays de l'Est de l'Europe une opportunité pour poursuivre leurs études à l'étranger. Aujourd'hui, de nombreux diplômés et cadres formés dans ces pays travaillent dans les administrations, les entreprises et le secteur des professions libérales. Mais les lauréats des pays de l'Est de l'Europe ont eu beaucoup de difficultés à se faire reconnaître auprès des élites marocaines plutôt formées dans les filières françaises d'excellence. Cet article aborde l'histoire de ces mobilités et le nouveau contexte migratoire qui la favorise. II vise à montrer qu'elles participent à la recomposition et à la diversification des destinations internationales des étudiants marocains. Si elles ont augmenté durant les années 1990 et 2000 les mobilités estudiantines marocaines ne sont pas uniquement dirigées vers la France. La filière migratoire marocaine d'études dans les pays de l'Est de l'Europe a connu un nouvel essor depuis la chute du mur de Berlin et elle renseigne sur la capacité d'adaptation des étudiants marocains face à l'évolution du contexte géopolitique des migrations d'étude.

\section{Moroccan Students' Training in Eastern Europe Countries (1960-2015)}

The Eastern Europe countries have been an ancient and rather particular component of the Moroccan foreign training system. Since early 1960s, the Moroccan students have found in the university system of Eastern Europe countries an excellent opportunity to study abroad. Nowadays many graduates and managerial staff, who were trained in those countries, work in administrations, enterprises, and liberal professions. However the graduates from the Eastern Europe countries have encountered obstacles to earn due acknowledgement, and insert thereafter the community of administrative and professional elite who has been trained in highly rated universities of France. This article deals with the history of this mobility and the new conducive migratory context. Though less in number compared to that of France, it has contributed to the recomposition and diversification of the international mobility of Moroccan students. Its increase during the 1990s and 2000s shows that the mobility of Moroccan students is not uniform despite the domination of France. The Moroccan migratory network of studies in Eastern Europe countries has witnessed a revival since the fall of Berlin wall. It displays Moroccan students' good ability of adaptation within a developing geopolitical context of migration related to studies. 


\section{La formación de los estudiantes marroquíes en los países de Europa del Este (1960-2015)}

La formación de los estudiantes marroquíes en los países de Europa del Este es un elemento muy particular y muy antiguo del sistema educativo marroquí en el extranjero. Desde principios de los años 1960, los estudiantes marroquíes han encontrado en los sistemas universitarios de los países de Europa del Este una gran oportunidad para estudiar en el extranjero. Hoy en día muchos titulados y directivos formados en estos países integran administraciones, empresas y profesiones liberales. Pero los graduados de estos países tuvieron muchas dificultades para ser reconocidos por las élites administrativas y profesionales marroquíes, a menudo formadas en las ramas de especialización francesas. Sin intentar proponer análisis definitivos, este articulo aborda la historia de esta movilidad y el nuevo contexto migratorio que la promueve. Su objetivo es mostrar que esta movilidad, aunque es menos importante que la llevada a cabo hacia Francia, comparte la composición de las movilidades internacionales de los estudiantes marroquíes y su diversificación. Aunque las movilidades de los estudiantes marroquíes se intensificaron durante los años 1990 y 2000, no se dirigieron únicamente hacia Francia. El flujo migratorio de los estudiantes marroquíes en los países de Europa del Este cobró auge desde la caída del muro de Berlín. Demuestra la capacidad de adaptación de los estudiantes marroquíes frente a la evolución del contexto geopolítico de las migraciones por motivos de estudios. 